\title{
Interaction of Edge Dislocations with Graphene Nanosheets in Graphene/Fe Composites
}

\author{
Lu Wang ${ }^{1}$, Jianfeng Jin ${ }^{1,2, *}$, Jingyi Cao ${ }^{1}$, Peijun Yang ${ }^{1}$ and Qing Peng ${ }^{3, *}$ \\ 1 School of Materials Science and Engineering \& Key Laboratory for Anisotropy and Texture of Materials, \\ Ministry of Education, Northeastern University, Shenyang 110819, China; wangl_edu@163.com (L.W.); \\ neu_caojy@163.com (J.C.); yangpj_yangcx@163.com (P.Y.) \\ 2 Northeastern Institute of Metal Materials Co., Ltd., Shenyang 110108, China \\ 3 Nuclear Engineering and Radiological Sciences, University of Michigan, Ann Arbor, MI 48109, USA \\ * Correspondence: jinjf@atm.neu.edu.cn (J.J.); qpeng@umich.edu (Q.P.)
}

Received: 1 February 2018; Accepted: 1 April 2018; Published: 4 April 2018

\begin{abstract}
Graphene is an ideal reinforcement material for metal-matrix composites owing to its exceptional mechanical properties. However, as a 2D layered material, graphene shows highly anisotropic behavior, which greatly affects the mechanical properties of graphene-based composites. In this study, the interaction between an edge dislocation $(\mathbf{b}=1 / 2(111))$ and a pair of graphene nanosheets (GNSs) in GNS reinforced iron matrix composite (GNS/Fe) was investigated using molecular dynamic simulations under simple shearing conditions. We studied the cases wherein

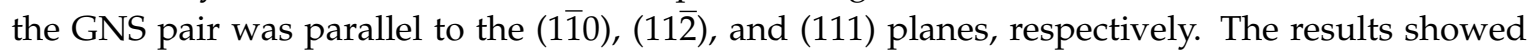
that the GNS reinforcement can effectively hinder dislocation motion, which improves the yield strength. The interaction between the edge dislocation and the GNS pair parallel to the $(11 \overline{2})$ plane showed the strongest effect of blocking dislocations among the three cases, resulting in increases in the shear modulus and yield stress of $107 \%$ and $1400 \%$, respectively. This remarkable enhancement was attributed to the Orowan "by-passing" strengthening mechanism, whereas cross-slip of dislocation segments was observed during looping around GNSs. Our results might contribute to the development of high-strength iron matrix composites.
\end{abstract}

Keywords: graphene; graphene-based iron matrix composite; mechanical properties; edge dislocation

\section{Introduction}

Graphene was firstly fabricated and identified as a single 2D carbon sheet with the same structure as individual layers of graphite by Novoselov and Geim in 2004 [1]. Graphene comprises a monolayer of carbon atoms arranged in a hexagonal lattice. It is an extremely stiff material with an intrinsic strength of $130 \mathrm{GPa}$ and Young's modulus of 1.0 TPa [2] and many other excellent physical and chemical properties [3,4]. These features make it attractive to be used in a vast number of applications. In particular, graphene has great potential to be used as a reinforcement material for enhancing the strength of graphene-based composites [5,6].

Many previous studies have focused on designing and fabricating new graphene reinforced metal matrix composites, including aluminum [7-9], copper [10,11], magnesium [12], and nickel matrix composites $[13,14]$. In most cases, it was observed that the mechanical properties were significantly enhanced, even with the addition of a very small amount of graphene. The tensile strength of a $0.3 \mathrm{wt} \%$ graphene nanosheet (GNS)-reinforced $\mathrm{Al}$ composite increased by $62 \%$ compared with that of the unreinforced matrix [9]. The elastic modulus and yield strength of graphene/copper nanocomposites containing $2.5 \mathrm{vol} \%$ reduced graphene oxide were $131 \mathrm{GPa}$ and $284 \mathrm{MPa}$, which were 1.3 and 1.8 times higher, respectively, compared with those of pure $\mathrm{Cu}$ [11]. Kim et al. synthesized a metal-graphene 
nanolayered composite and found that the addition of GNS resulted in an ultra-high strength of 4.0 GPa for a graphene/nickel composite [14]. Moreover, the elastic modulus and yield strength of a copper matrix composite reinforced with GNS and Ni nanoparticle hybrids increased $61 \%$ and $94 \%$, respectively, compared with the pure matrix, for a GNS content of only $1.0 \mathrm{vol} \%$ [15]. As a 2D layered material, graphene shows highly anisotropic behavior, which greatly affects the mechanical properties of graphene-based composites. For example, the microstructures and anisotropic tensile properties of graphene nanoplatelets (GNP) reinforced copper composites with 10 and $20 \mathrm{vol} \%$ of GNPs were studied experimentally, where a fairly good GNP alignment was achieved in the composites, leading to the prominent anisotropic mechanical properties with in-plane tensile strength and elongation significantly outperforming through-plane ones [16].

The exploration of applications of graphene as a reinforcement material is still in its infancy. For example, the effects of graphene reinforcement of iron on the properties of the corresponding composites are not yet well understood. To gain a fundamental understanding the role of graphene in graphene-based metal composites during deformation, the molecular dynamics (MD) method has been widely used in recent years to study strengthening mechanisms at the atomic scale. The MD results showed that graphene effectively blocks dislocation propagation across the graphene-Ni interface [14]. Shi et al. used MD to study the binding, peeling, and folding of graphene on a single crystal $\mathrm{Cu}$ surface, where the graphene had the highest binding energy on the $\mathrm{Cu}$ (111) surface, followed by the (100), (110), and (112) surfaces [17]. Moreover, $\mathrm{Xu}$ and Buehler used density functional theory to study the binding energy, bonding strength, and electronic structure of graphene on the $\mathrm{Cu}$ (111) surface [18]. Both studies provided a preliminary understanding of the interfacial mechanical behavior of graphene/Cu nanocomposites. The effect of Ni-coating on the mechanical behaviors of single GNSs and their corresponding Al-matrix composites under axial tension was investigated using MD simulations; the Young's modulus and tensile strength of the Ni-coated graphene/ Al composite were clearly higher than those of the composite with uncoated reinforcement and those of the pure matrix material [19].

There exist a few studies using BCC metal as the matrix in graphene-based composites. The aim of this work is to explore the mechanical behavior of GNS-reinforced BCC iron matrix composites using MD simulations. In practice, the single-layer graphene oxide (GO) reinforced iron matrix nanocomposite has been successfully fabricated using a laser-based additive manufacturing process to sinter GO and iron powders [20]. One of the key factors in increasing the strength of the graphene metal composite is to effectively hinder dislocation motion. Therefore, we will focus on the interaction between an edge dislocation and a pair of single-layer graphene nanosheets (GNSs) located at different crystal planes. The mechanical response of the graphene/Fe composite is investigated during deformation and is discussed with respect to dislocation evolution.

\section{Model Setup and Interatomic Potential}

For atomic level understanding of dislocation-GNS interactions, the MD method was employed. For numerical implementation of the MD simulations, we used the large-scale atomic/molecular massively parallel simulator (LAMMPS) software [21]. A schematic diagram of the simulation cell in three dimensions is shown in Figure 1a. The periodic boundary condition was applied to the two in-plane directions ( $x$ and $y$ ) but not to the out-of-plane direction ( $\mathrm{z}$ ). Two pistons at the top and the bottom of the simulation cell, each consisting of three (11) atom layers, mimicked a large bulk environment. Because the strain $\left(\varepsilon_{\mathrm{yz}}\right)$ was gradually increased at every integration step, the increment was applied to only the top piston but not the interior of the simulation cell. We introduced an edge dislocation on the shaded $(1 \overline{1} 0)$ plane by removing three adjacent (111) layers of atoms above this ( $(1 \overline{1} 0)$ glide plane. The Burgers vector was $1 / 2$ (111). The dislocation density in the simulation cell was about $5 \times 10^{9} / \mathrm{m}^{2}$. The simulation cell was relaxed, where the dimensions were changed to minimize the magnitude of all stress components, according to the Parrinello-Rahman algorithm [22]. 


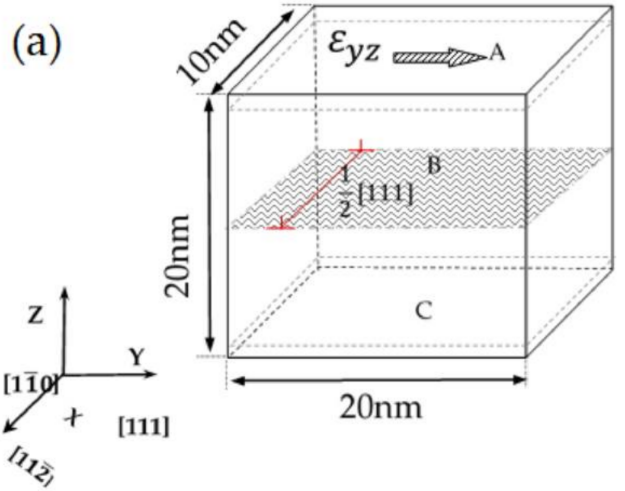

(b)

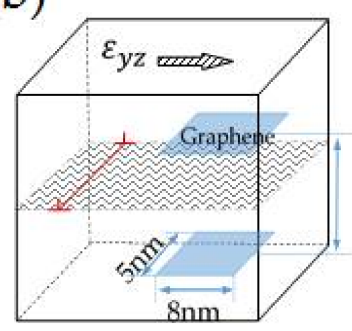

GNS $/ /(1 \overline{1} 0)$

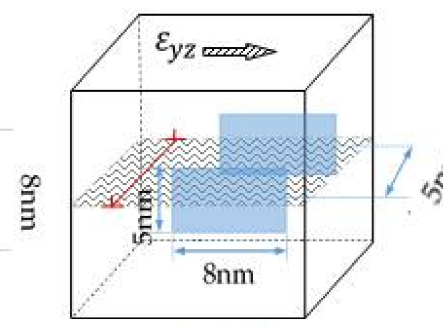

GNS//(11̄)

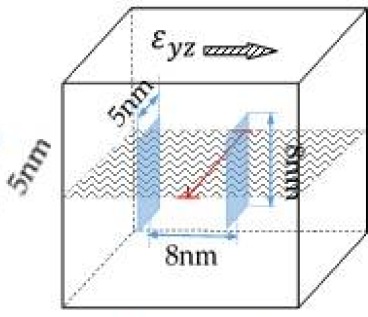

GNS//(111)

Figure 1. Schematic of (a) the simulation cell in three dimensions and (b) the graphene nanosheet (GNS) pair in a graphene/Fe composite aligned along three different Fe crystal planes.

We introduced a pair of single-layer GNSs with an area of $8 \mathrm{~nm} \times 5 \mathrm{~nm}$ into a BCC $\alpha$-Fe matrix with a lattice constant of $2.85 \AA$ by removing one layer of Fe atoms in the same area, where the GNS content was $0.7 \mathrm{vol} \%$. The size and volume fraction picked here is not far from reality, which based on the other references, wherein the graphene size was chosen to $2.02 \times 4.25 \mathrm{~nm}^{2}$ for graphene sheet [19] and 4-12 nm for graphene nanoribbons [23] for the MD simulations, and from experimental results, the volume fraction of graphene reinforcement was $1.13 \mathrm{vol} \%$ in GO reinforced iron matrix composites [20] and $0.2 \mathrm{vol} \%$ in graphene-based $\mathrm{Al}$ composites [7]. Comparing the system potential energy values after removing 1-3 layers of iron atoms, the total energy was the lowest when the GNS was added after removing only one layer of iron atoms, associated with the atomic structures, listed in Table 1. Then, we set up three configurations of the graphene location, normal to the $\mathrm{x}, \mathrm{y}$, and $\mathrm{z}$ directions, as shown in Figure 1b.

The selection of interatomic potential is a key factor determining the accuracy of MD simulation results. The Brenner second-generation reactive empirical bond-order potential $[24,25]$ was used to model the $\mathrm{C}-\mathrm{C}$ bounded interaction, while the $\mathrm{Fe}-\mathrm{Fe}$ and $\mathrm{Fe}-\mathrm{C}$ interactions were modeled with the embedded atom potential [26]. The embedded atom potential is described by two terms [27]:

$$
\mathrm{E}_{\mathrm{M}-\mathrm{X}}=\mathrm{F}_{\alpha}\left(\Sigma_{\mathrm{j} \neq \mathrm{i}} \rho_{\beta}\left(\mathrm{r}_{\mathrm{ij}}\right)\right)+\frac{1}{2} \Sigma_{\mathrm{j} \neq \mathrm{i}} \varnothing_{\alpha \beta}\left(\mathrm{r}_{\mathrm{ij}}\right),
$$

where $F_{\alpha}$ is the embedding energy as a function of the electron density $\rho\left(r_{i j}\right)$ of the atom $j, \varnothing_{\alpha \beta}$ is a pair potential interaction, and $\alpha$ and $\beta$ are the element types of atoms $i$ and $j$, respectively. The $C-C$ AIREBO potential [28] is represented by three terms:

$$
\mathrm{E}=\frac{1}{2} \Sigma_{\mathrm{i}} \Sigma_{\mathrm{j} \neq \mathrm{i}}\left[\mathrm{E}_{\mathrm{ij}}^{\mathrm{REBO}}+\mathrm{E}_{\mathrm{ij}}^{\mathrm{L}-\mathrm{J}}+\Sigma_{\mathrm{k} \neq \mathrm{i}, \mathrm{j}} \Sigma_{\mathrm{l} \neq \mathrm{i}, \mathrm{j}, \mathrm{k}} \mathrm{E}_{\mathrm{kij}}^{\mathrm{TORSION}}\right],
$$

where the $\mathrm{E}_{\mathrm{ij}}^{\mathrm{REBO}}$ term [24] takes the form of the hydrocarbon REBO potential to only describe short-ranged C-C interactions $\left(\mathrm{r}<2 \AA\right.$ ), which gives the model reactive capability. The $\mathrm{E}_{\mathrm{ij}}^{\mathrm{L}-\mathrm{J}}$ term 
uses the form of a Lennard-Jones potential for longer-range interactions ( $2 \AA<r<r_{\text {cutoff }}$ ), while the $\mathrm{E}_{\mathrm{kij}}^{\mathrm{TORSION}}$ term is an explicit 4-body potential that describes various dihedral angle preferences in hydrocarbon configurations. The C-C AIREBO potential has been widely used in MD simulations for graphene-based materials [19,29].

Then, we applied a shear strain $\left(\varepsilon_{\mathrm{yz}}\right)$ with a strain rate of $2.5 \times 10^{7} / \mathrm{s}$ and kept the temperature at room temperature of $300 \mathrm{~K}$ using the Nose-Hoover algorithm [30]. A small and equal strain increment of $1.25 \times 10^{-4}$ was applied every 5000-integration step (5 ps). For each strain step, we calculated the stress as the average atomic level viral stress [31] in the dynamic region only (i.e., excluding the piston regions). The common neighbor analysis was used to identify defect atoms [32], such as dislocation cores and surface atoms, and the OVITO software was used to visualize the atomic structure [33]. The effective stress was calculated using:

$$
\bar{\sigma}=\sqrt{\frac{1}{2}\left[\left(\sigma_{x x}-\sigma_{y y}\right)^{2}+\left(\sigma_{y y}-\sigma_{z z}\right)^{2}+\left(\sigma_{x x}-\sigma_{z z}\right)^{2}\right]+3\left(\sigma_{x y}^{2}+\sigma_{x z}^{2}+\sigma_{y z}^{2}\right)}
$$

where $\sigma_{x x}, \sigma_{y y}, \sigma_{z z}, \sigma_{x y}, \sigma_{x z}$ and $\sigma_{y z}$ are the six components of an atomic level stress. Here, the molecular static and dynamic simulations with a higher strain rate of $2.5 \times 10^{8} / \mathrm{s}$ were also tested. In the $2.5 \times 10^{7} / \mathrm{s}$ case, the yield stress was lower than that in the static case and close to that in the $2.5 \times 10^{8} / \mathrm{s}$ one. For the same GNS location, dislocation responses in the three cases were very close, which proved that dislocation responses in a lower strain rate case would be the same.

Table 1. Comparison of the atomic structure and the total energy $\mathrm{E}_{\text {total }}=\mathrm{E}_{\mathrm{GNS} / \mathrm{Fe}}+\mathrm{N}_{\mathrm{Fe}}^{\mathrm{Remove}} \times$ $\mathrm{E}_{\mathrm{Fe}}^{\text {Cohesive }}$ with respect to the GNS locations, where $\mathrm{E}_{\mathrm{GNS} / \mathrm{Fe}}$ is the minimized potential energy of the GNS/Fe composite after removing 1-3 layers of iron atoms, $\mathrm{N}_{\mathrm{Fe}}^{\mathrm{Re}}$ ive is the number of removed iron atoms and $\mathrm{E}_{\mathrm{Fe}}^{\text {Cohesive }}$ is the cohesive energy of iron.

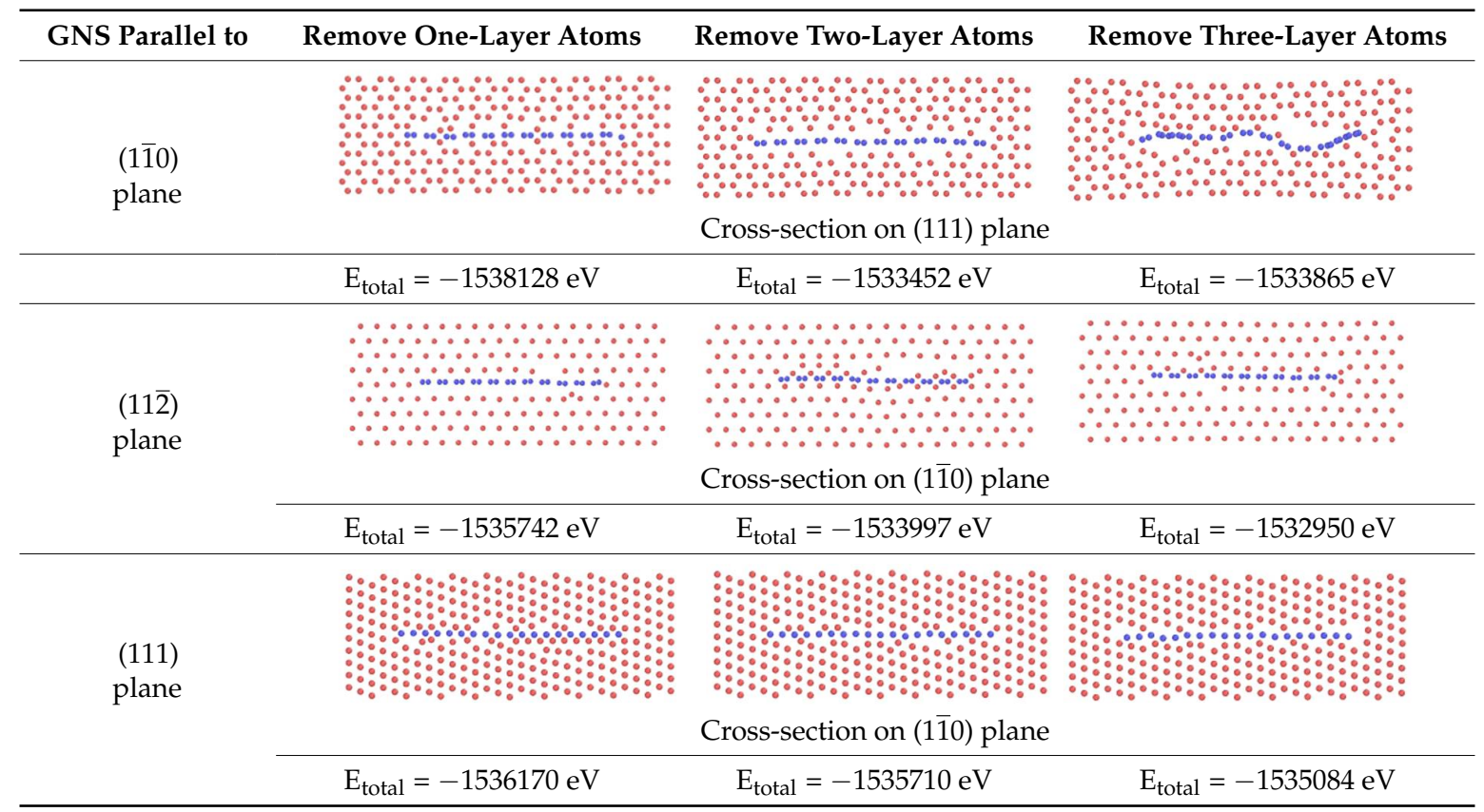

\section{Results and Discussion}

We focused on the stress-strain response of the GNS/Fe composite reinforced by GNS pairs at different location with a GNS content of $0.7 \mathrm{vol} \%$ during deformation and observed the dislocation evolution. In particular, we studied the non-isotropic mechanical behavior along three typical

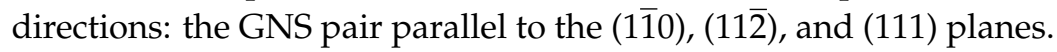




\subsection{Interaction between the Edge Dislocation and GNS Pair Parallel to the (1̄̄0) Plane}

The stress-strain curves from the MD simulations are shown in Figure 2a for two cases, with and without the GNS pair in the iron matrix. The GNS pairs considered here were parallel to the (110) plane. The red curve corresponds to a single edge dislocation in the iron matrix without GNS reinforcement. The shear modulus was about $56.4 \mathrm{GPa}$, lower than previous MD results showing $\sim 60 \mathrm{GPa}$ [34], which may be related to different iron potentials or a smaller simulation cell in our case. Dislocation motion needs to overcome the Peierls-Nabarro barrier. In this study, the yield stress for dislocation movement was $110 \mathrm{MPa}$, which was higher than other previously published potential values of 25 and $90 \mathrm{MPa}$ [34], and the stress was observed to swing during dislocation motion to overcome the barrier in periodic-arranged crystal lattices. From the stress-strain curve of the GNS/Fe composite with the GNS parallel to the (110) plane, as shown in Figure 2a, we determined shear modulus and yield stress values of $86.6 \mathrm{GPa}$ and $479.9 \mathrm{MPa}$, respectively, which were an increase of $54 \%$ and $336 \%$, respectively, compared to the unreinforced iron.
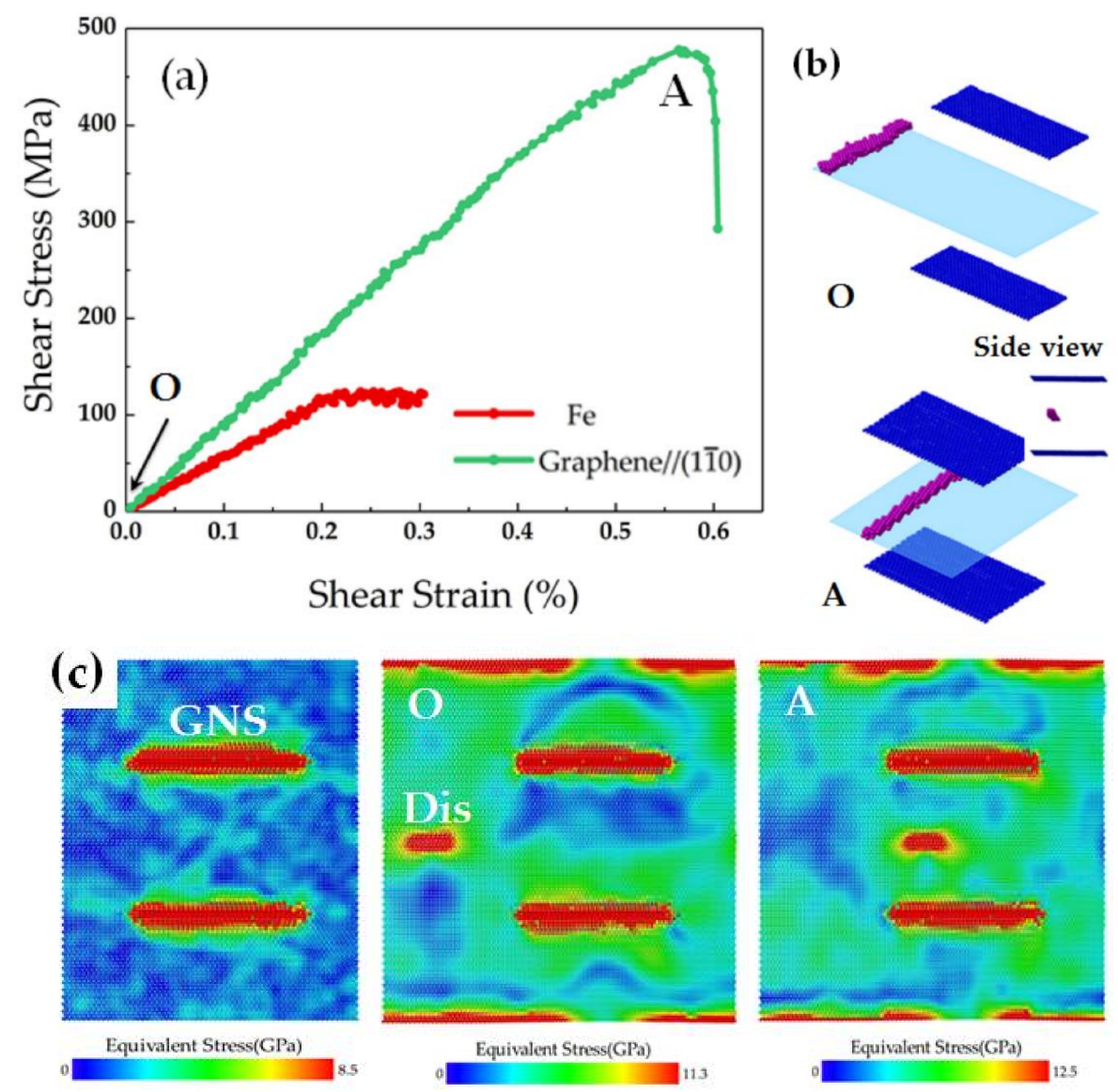

Figure 2. (a) Stress-strain curves of the pure iron and GNS/Fe composite (GNS//(11)0)) with an edge dislocation; (b) dislocation morphology at the points $\mathrm{O}$ and $\mathrm{A}$ in the stress-strain curve shown in (a) (blue: GNS; pink: dislocation core; and shaded plane: gliding plane); and (c) effective stress contours for the GNS/Fe composite without dislocation before deformation and with dislocation at points $\mathrm{O}$ and $\mathrm{A}$.

To understand yielding in these materials, we studied dislocation and GNS pair interactions at the atomic level and observed no apparent change in the dislocation morphology (Figure 2b). The contours of the effective stress ( $\bar{\sigma})$ calculated from Equation (3) for the GNS/Fe composite without a dislocation before deformation and with a dislocation during deformation are shown in Figure 2c. It can be seen that after the GNS was embedded into the iron matrix, a local stress field produced by the lattice mismatch of the GNS. As the shearing direction was parallel to the GNS plane, the GNS reinforcement 
bore some load through interfacial friction, resulting to the increase of yield stress, and the change in the local stress field in the GNS/Fe composite during shearing was also shown in Figure 2c, where the load bearing capability of the GNS reinforcement reached the maximum value at the point A. The strengthening mechanism in this case was mainly load transfer (i.e., direct strengthening) rather than the enhancing matrix (or indirect strengthening).

\subsection{Interaction between the Edge Dislocation and GNS Pair Parallel to the (11̄) Plane}

From the stress-strain curve of the GNS/Fe composite with the GNS parallel to the (112) plane shown in Figure 3a, it can be seen that there were two different regions: the OA and AE stages.

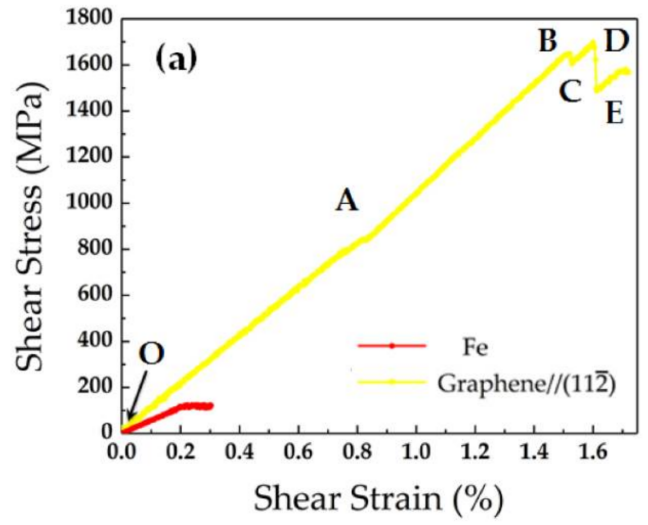

Front view Top view (b)
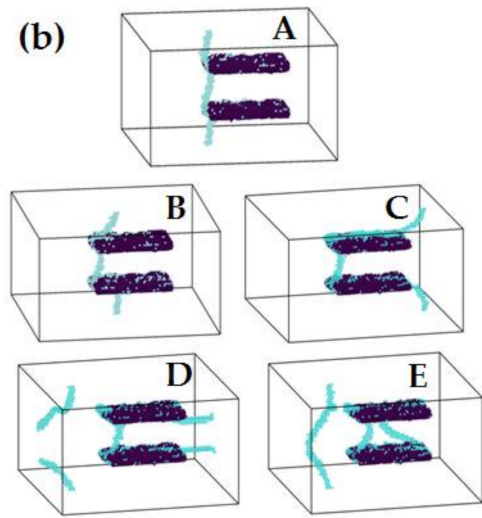

Front view Top view

\section{(c)}
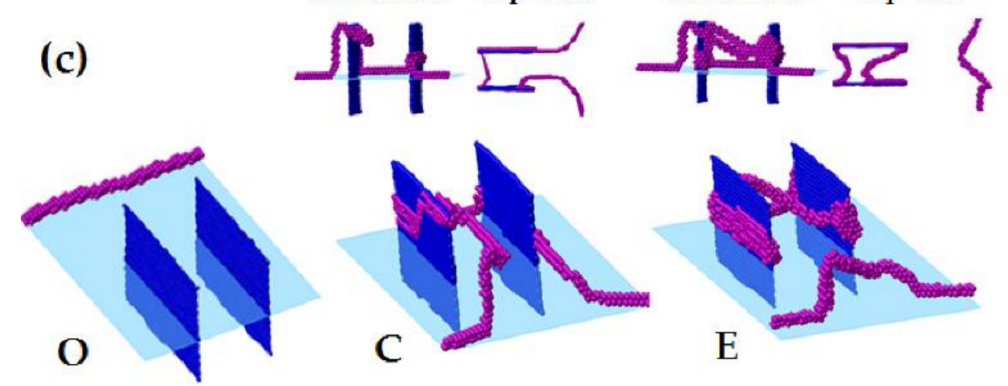

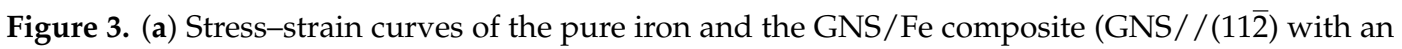
edge dislocation inside; (b) dislocation morphology at the points indicated on the stress-strain curve showed in (a); (c) detailed dislocation morphology at the relevant points (blue: GNS; pink: dislocation core; and shaded plane: gliding plane).

During the OA stage, the dislocation started to move and approached the GNS pair at point A (as shown in Figure 3b), where the corresponding shear modulus and first yield stress were 108.6 GPa and $836.6 \mathrm{MPa}$, increases of $93 \%$ and $660 \%$, respectively compared to the unreinforced case. After further loading, the stress-strain curve moved to the AE stage and further yielding occurred. Unlike conventional nanoscale ductile metal particles in metal-matrix composites, the stiff GNS cannot be sheared via dislocations, due to the high strength of $\mathrm{C}-\mathrm{C}$ bonding. The shear modulus and the second yield stress values were 116.7 GPa and 1573.2 MPa, respectively, corresponding to increases of $107 \%$ and $1330 \%$, respectively, compared to the non-reinforced case. It can be seen in Figure $3 b, c$ that the dislocation line partially covered the GNS pair surface at point C. Cross-slip behavior was observed, which cannot occur in edge dislocations. However, when the dislocation line attached the sides of the GNS, it became parallel to the Burgers vector $\mathbf{b}$ and screw segments were formed, leading to cross-slip and the second minor yield point. Then, a third yielding occurred with a stress of $1647.3 \mathrm{MPa}$, where the dislocation de-pinned from the GNS, leaving a loop around the GNS pair surface; this corresponded to point $\mathrm{E}$ in Figure 3b,c. This phenomenon was related to Orowan "by-passing" mechanism. At 
this point, the depinning stress of the GNS/Fe composite was fourteen times higher than that of unreinforced iron. Our results confirmed that the reinforcement effect of graphene in iron is very effective. After the dislocation bypassed the GNS pair, it continued in forward motion and crossed the periodic boundary, and then approached the GNS pair for the second time. In order to make the dislocation structure clear, we showed the depinned dislocation line in front, rather than crossing the boundary, at point $\mathrm{E}$ in Figure 3c.

\subsection{Interaction with the Edge Dislocation and GNS Pair Parallel to the (111) Plane}

The stress-strain curve of the GNS/Fe composite with the GNS parallel to the (111) plane is shown in Figure 4a. Like the former case, this curve also showed two stages: the OA and AC stages. Similar to the previous case, in the OA stage, the dislocation started to move and approach the GNS pair at point $\mathrm{A}$ in Figure $4 \mathrm{~b}$. The shear modulus and first yield stress were $71.1 \mathrm{GPa}$ and $170.5 \mathrm{MPa}$, respectively, corresponding to increases of $26 \%$ and $55 \%$, respectively, compared to those of the unreinforced iron. After further loading, the stress-strain curve moved into the AC stage and further yielding occurred. The shear modulus and second yield stress (depinning stress) were $103.3 \mathrm{GPa}$ and $1401 \mathrm{MPa}$, corresponding to increases of $83 \%$ and $1173 \%$, respectively compared to the non-reinforced case. The Orowan "by-passing" interaction mechanism was still relevant at point $C$ in Figure 4 b,c. No cross-slip of the dislocation was observed due to the lack of screw segments. However, dislocation climbing was observed in the dislocation loop around the GNS, which may have been caused by stress concentration around the GNS. Furthermore, as the length of the dislocation loop in this case was about $10 \mathrm{~nm}$ (i.e., two times the $5 \mathrm{~nm}$ width of the GNSs), which was shorter than that of $26 \mathrm{~nm}$ (i.e., two times the GNS pair interspacing of $5 \mathrm{~nm}$ and GNS width of $8 \mathrm{~nm}$ ) in the GNS//(112) case, the corresponding depinning stress was lower in the latter case.

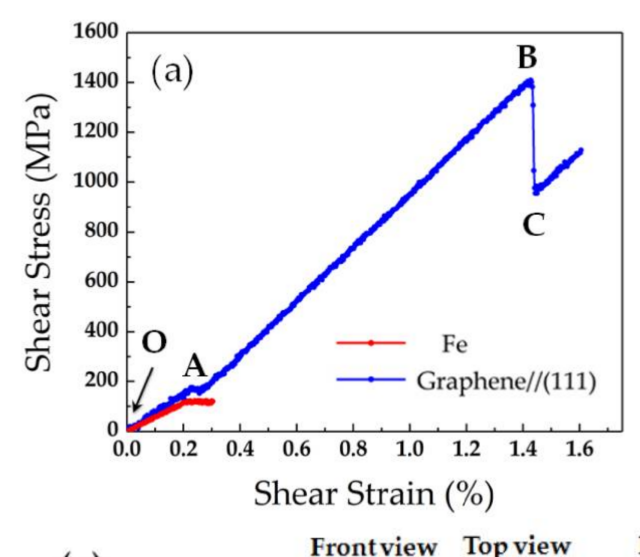

(b)
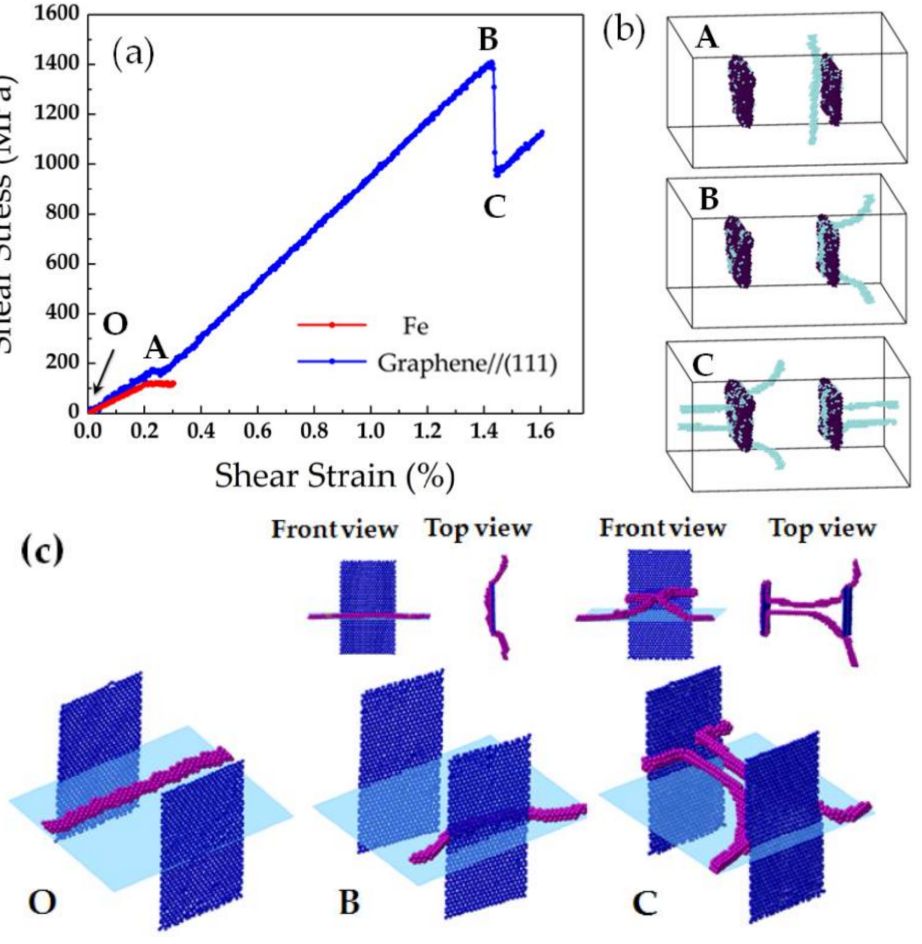

Figure 4. (a) Stress-strain curves of pure iron and the GNS/Fe composite (GNS//(111)) with an edge dislocation inside; (b) dislocation morphology at the points indicated on the stress-strain curve showed in (a); (c) detailed dislocation morphology at the relevant points (blue: GNS; pink: dislocation core; and shaded plane: gliding plane). 
It is clear that the GNS pair provided an efficient barrier to dislocation motion in the GNS/Fe composite. This dislocation strengthening mechanism was anisotropic. Comparing the MD results for the three GNS pair locations listed in Table 2, the interaction of the edge dislocation and GNS pair parallel to the $(11 \overline{2})$ plane had the strongest blocking effect, resulting in an ultra-high strength of the graphene/Fe composite of $1647.3 \mathrm{MPa}$, which was about 14 times higher than that of the unreinforced case. The lowest reinforcement behavior was observed for the GNS placed along the (11)0) plane. The average value of the depinning stress of all the samples herein was $1176 \mathrm{MPa}$.

Table 2. Comparison of shear modulus and yield or depinning stress of GNS/Fe composites reinforced by GNS pairs aligned along three different Fe crystal planes.

\begin{tabular}{ccccccc}
\hline & \multirow{2}{*}{ Fe } & \multirow{2}{*}{ GNS//(11) } & \multicolumn{2}{c}{ GNS//(112) } & \multicolumn{2}{c}{ GNS//(111) } \\
\cline { 3 - 6 } & & & Approach & Depin & Approach & Depin \\
\hline Shear Modulus $(\mathrm{GPa})$ & 56.4 & 86.6 & 108.6 & 116.7 & 71.1 & 103.3 \\
Yield Stress $(\mathrm{MPa})$ & 110 & 479.9 & 836.6 & 1647.3 & 170.5 & 1401 \\
\hline
\end{tabular}

Our results provide a first approximation of the reinforcement behavior of graphene in iron or steel composites. One might expect that a higher concentration of graphene further increases the dislocation migration barrier crossing the graphene. Our results qualitatively agree with previous studies of other particle-reinforced metal-matrix composites, such as $\mathrm{SiCp} / \mathrm{Cu}$ [35]. While using GNS to enhance the mechanical properties of the iron matrix has demonstrated great success, further research is necessary. It is expected that the level of enhancement could be further increased by optimizing the size, volume fraction, and orientation of graphene-based reinforcement materials. Furthermore, the simulated yield or depinning stress of the GNS/Fe composites with respect to the GNS location may also be regarded as a critical stress, which could be used as input parameters for a large-scale model. Similar strategy has been used successfully in [36], where dislocation-precipitate interactions in Fe was studied by multiscale modeling and the parameters used in the discrete dislocation dynamics simulations for the dislocation mobility, shear modulus, and dislocation core energy were obtained from MD simulations.

\section{Conclusions}

We investigated the interaction of an edge dislocation with a pair of GNSs in graphene/Fe composites using MD simulations and have drawn the following conclusions.

1. It is clear that the GNSs provide an efficient barrier to dislocation motion in the GNS/Fe composite.

2. When the GNS was parallel to the (110) plane, the shear modulus and yield stress was $86.6 \mathrm{GPa}$ and $479.9 \mathrm{MPa}$, an increase of $54 \%$ and $336 \%$, respectively, compared to the unreinforced matrix.

3. When the GNS was parallel to the $(11 \overline{2})$ plane, the shear modulus and yield stress for the dislocation-GNS interaction was $116.7 \mathrm{GPa}$ and $1647.3 \mathrm{MPa}$, an increase of $107 \%$ and $1400 \%$, respectively, compared to the unreinforced case, where the Orowan "by-passing" strengthening mechanism was observed.

4. When the GNS was parallel to the (111) plane, the shear modulus and yield stress for the interaction were $103.3 \mathrm{GPa}$ and $1401 \mathrm{MPa}$, an increase of $83 \%$ and $1173 \%$, respectively, compared to the unreinforced iron. The Orowan "by-passing" mechanism was also observed.

5. Comparing the mechanical behavior of the iron matrix composite reinforced by GNS pairs located at different places in the iron matrix, the interaction of the edge dislocation and GNS pair parallel to the $(11 \overline{2})$ plane had the strongest blockage effect, resulting in an ultra-high strength of the respective graphene/Fe composite. 
Acknowledgments: Authors would like to acknowledge the National Key Research and Development Program of China (No. 2016YFB0701204), the Projects by National Natural Science Foundation of China (No. U1302272, 51301035), National Higher-education Institution General Research and Development Project (No. N151004004, N150502002) and Program for Liaoning Excellent Talents in University (No. LJQ2015037).

Author Contributions: Lu Wang, Jianfeng Jin and Qing Peng conceived and designed the simulations; Lu Wang, Jingyi Cao and Peijun Yang performed the simulations; Lu Wang, Jianfeng Jin and Qing Peng analyzed the data; Jianfeng Jin and Qing Peng wrote the paper. All authors discussed and commented on the manuscript.

Conflicts of Interest: The authors declare no conflict of interest.

\section{References}

1. Novoselov, K.S.; Geim, A.K.; Morozov, S.V.; Jiang, D.; Zhang, Y.; Dubonos, S.V.; Grigorieva, I.V.; Firsov, A.A. Electric field effect in atomically thin carbon films. Science 2004, 306, 666-669. [CrossRef] [PubMed]

2. Lee, C.; Wei, X.; Kysar, J.W.; Hone, J. Measurement of the elastic properties and intrinsic strength of monolayer graphene. Science 2008, 321, 385-388. [CrossRef] [PubMed]

3. Novoselov, K.S. Graphene: Materials in the Flatland (Nobel lecture). Angew. Chem. Int. Ed. 2011, 50, 6986-7002. [CrossRef] [PubMed]

4. Peng, Q.; Liang, C.; Ji, W.; De, S. A theoretical analysis of the effect of the hydrogenation of graphene to graphane on its mechanical properties. Phys. Chem. Chem. Phys. 2013, 15, 2003-2011. [CrossRef] [PubMed]

5. Young, R.J.; Kinloch, I.A.; Gong, L.; Novoselov, K.S. The mechanics of graphene nanocomposites: A review. Compos. Sci. Technol. 2012, 72, 1459-1476. [CrossRef]

6. Papageorgiou, D.G.; Kinloch, I.A.; Young, R.J. Mechanical properties of graphene and graphene-based nanocomposites. Prog. Mater. Sci. 2017, 90, 75-127. [CrossRef]

7. Bartolucci, S.F.; Paras, J.; Rafiee, M.A.; Rafiee, J.; Lee, S.; Kapoor, D.; Koratkar, N. Graphene-aluminum nanocomposites. Mater. Sci. Eng. A 2011, 528, 7933-7937. [CrossRef]

8. Jeon, C.H.; Jeong, Y.H.; Seo, J.J.; Tien, H.N.; Hong, S.T.; Yum, Y.-J.; Hur, S.-H.; Lee, K.-J. Material properties of graphene/aluminum metal matrix composites fabricated by friction stir processing. Int. J. Precis. Eng. Manuf. 2014, 15, 1235-1239. [CrossRef]

9. Wang, J.Y.; Li, Z.Q.; Fan, G.L.; Pan, H.H.; Chen, Z.X.; Zhang, D. Reinforcement with graphene nanosheets in aluminum matrix composites. Scr. Mater. 2012, 66, 594-597. [CrossRef]

10. Pavithra, C.L.; Sarada, B.V.; Rajulapati, K.V.; Sundararajan, T.N.R.G. A new electrochemical approach for the synthesis of copper-graphene nanocomposite foils with high hardness. Sci. Rep. 2014, 4, 4049. [CrossRef] [PubMed]

11. Hwang, J.; Yoon, T.; Jin, S.H.; Lee, J.S.; Kim, T.-S.; Hong, S.H.; Jeon, S. Enhanced mechanical properties of graphene/copper nanocomposites using a molecular-level mixing process. Adv. Mater. 2013, 25, 6724-6729. [CrossRef] [PubMed]

12. Chen, L.Y.; Konishi, H.; Fehrenbacher, A.; Ma, C.; Xu, J.-Q.; Choi, H.; Xu, H.-F.; Pfefferkorn, F.E.; Li, X.-C. Novel nanoprocessing route for bulk graphene nanoplatelets reinforced metal matrix nanocomposites. Scr. Mater. 2012, 67, 29-32. [CrossRef]

13. Yazdandoost, F.; Boroujeni, A.Y.; Mirzaeifar, R. Nanocrystalline nickel-graphene nanoplatelets composite: Superior mechanical properties and mechanics of properties enhancement at the atomistic level. Phys. Rev. Mater. 2017, 1, 076001. [CrossRef]

14. Kim, Y.; Lee, J.; Yeom, M.S.; Shin, J.W.; Kim, H.; Cui, Y.; Kysar, J.W.; Hone, J.; Jung, Y.; Jeon, S.; et al. Strengthening effect of single-atomic-layer graphene in metal-graphene nanolayered composites. Nat. Commun. 2013, 4, 2114. [CrossRef] [PubMed]

15. Tang, Y.X.; Yang, X.M.; Wang, R.R.; Li, M. Enhancement of the mechanical properties of graphene-copper composites with graphene-nickel hybrids. Mater. Sci. Eng. A 2014, 599, 247-254. [CrossRef]

16. Chu, K.; Wang, F.; Wang, X.; Huang, D.-J. Anisotropic mechanical properties of graphene/copper composites with aligned graphene. Mater. Sci. Eng. A 2018, 713, 269-277. [CrossRef]

17. Shi, X.H.; Yin, Q.F.; Wei, Y.J. A theoretical analysis of the surface dependent binding, peeling and folding of graphene on single crystal copper. Carbon 2012, 50, 3055-3063. [CrossRef]

18. Xu, Z.; Buehler, M.J. Interface structure and mechanics between graphene and metal substrates: A first-principles study. J. Phys. Condens. Matter 2010, 22, 485301. [CrossRef] [PubMed] 
19. Song, H.Y.; Zha, X.W. Mechanical properties of Ni-coated single graphene sheet and their embedded aluminum matrix composites. Commun. Theor. Phys. 2010, 54, 143.

20. Lin, D.; Liu, C.; Cheng, G. Single-layer graphene oxide reinforced metal matrix composites by laser sintering: Microstructure and mechanical property enhancement. Acta Mater. 2014, 80, 183-193. [CrossRef]

21. Plimpton, S. Fast parallel algorithms for short-range molecular-dynamics. J. Comput. Phys. 1995, 117, 1-19. [CrossRef]

22. Parrinello, M.; Rahman, A. Polymorphic transitions in single crystals: A new molecular dynamics method. J. Appl. Phys. 1981, 52, 7182-7190. [CrossRef]

23. Chu, Y.; Ragab, T.; Basaran, C. The size effect in mechanical properties of finite-sized graphene nanoribbon. Comput. Mater. Sci. 2014, 81, 269-274. [CrossRef]

24. Brenner, D.W.; Shenderova, O.A.; Harrison, J.A.; Stuart, S.J.; Ni, B.; Sinnott, S.B. A second-generation reactive empirical bond order (REBO) potential energy expression for hydrocarbons. J. Phys. Condens. Matter 2002, 14, 783-802. [CrossRef]

25. Deng, B.; Hou, J.; Zhu, H.; Liu, S.; Liu, E.; Shi, Y.F.; Peng, Q. The normal-auxeticity mechanical phase transition in graphene. 2D Mater. 2017, 4, 2. [CrossRef]

26. Becquart, C.S.; Raulot, J.M.; Bencteux, G.; Domain, S.; Perez, M.; Garruchet, S.; Nguyen, H. Atomistic modeling of an Fe system with a small concentration of C. Comput. Mater. Sci. 2007, 40, 119-129. [CrossRef]

27. Daw, M.S.; Baskes, M.I. Embedded-atom method: Derivation and application to impurities, surfaces, and other defects in metals. Phys. Rev. B 1984, 29, 6443-6453. [CrossRef]

28. Stuart, S.J.; Tutein, A.B.; Harrison, J.A. A reactive potential for hydrocarbons with intermolecular interactions. J. Chem. Phys. 2000, 112, 6472-6486. [CrossRef]

29. Dewapriya, M.A.N.; Meguid, S.A. Atomistic modeling of out-of-plane deformation of a propagating Griffith crack in graphene. Acta Mech. 2017, 228, 3063-3075. [CrossRef]

30. Hoover, W.G. Canonical dynamics: Equilibrium phase-space distributions. Phys. Rev. A Gen. Phys. 1985, 31, 1695-1697. [CrossRef] [PubMed]

31. Zhou, M. A new look at the atomic level virial stress: On continuum-molecular system equivalence. Proc. $R$. Soc. A Math. Phys. Eng. Sci. 2003, 459, 2347-2392. [CrossRef]

32. Tsuzuki, H.; Branicio, P.S.; Rino, J.P. Structural characterization of deformed crystals by analysis of common atomic neighborhood. Comput. Phys. Commun. 2007, 177, 518-523. [CrossRef]

33. Stukowski, A. Visualization and analysis of atomistic simulation data with OVITO-The open visualization tool. Model. Simul. Mater. Sci. Eng. 2010, 18. [CrossRef]

34. Terentyev, D.; Bacon, D.J.; Osetsky, Y.N. Interaction of an edge dislocation with voids in alpha-iron modelled with different interatomic potentials. J. Phys. Condens. Matter 2008, 20, 445007. [CrossRef]

35. Li, J.; Liu, B.; Fang, Q.; Huang, Z.W.; Liu, Y.W. Atomic-scale strengthening mechanism of dislocation-obstacle interaction in silicon carbide particle-reinforced copper matrix nanocomposites. Ceram. Int. 2017, 43, 3839-3846. [CrossRef]

36. Lehtinen, A.; Granberg, F.; Laurson, L.; Nordlund, K.; Alava, M.J. Multiscale modeling of dislocation-precipitate interactions in Fe: From molecular dynamics to discrete dislocations. Phys. Rev. E 2016, 93, 013309. [CrossRef] [PubMed]

(C) 2018 by the authors. Licensee MDPI, Basel, Switzerland. This article is an open access article distributed under the terms and conditions of the Creative Commons Attribution (CC BY) license (http://creativecommons.org/licenses/by/4.0/). 